\title{
Lavendustin C
}

National Cancer Institute

\section{Source}

National Cancer Institute. Lavendustin C. NCI Thesaurus. Code C1487.

A compound that inhibits calmodulin kinase II and p60. ( $\mathrm{NCl})$ 